\title{
Superior Hypogastric Plexus Neurolysis to Manage Metastatic Pelvic Pain in a Forty-Five-Year-Old Spina Bifida Patient: A Case Report
}

\author{
Somnath Bagchia, b, e , Rosie Kemp ${ }^{\mathrm{c}}$, Christopher J. Green ${ }^{\mathrm{a}}$, Jeffrey Stephenson ${ }^{\mathrm{d}}$
}

\begin{abstract}
A 45-year-old patient with spina bifida and adenocarcinoma of the rectum was treated with a superior hypogastric plexus (SHP) ablation for pain control. The procedure enabled her to reduce opioid consumption, being more clear-headed and functional to be discharged to her residence. The case is presented to highlight the options of neurolytic interventions to manage pain in terminally ill cancer patients. We discuss the options of SHP ablation and justify our choice of approach and the use of a neurolytic agent.
\end{abstract}

Keywords: Cancer pain; Spina bifida; Neurolysis; Superior hypogastric plexus neurolytic block

\section{Introduction}

Pain is a common symptom affecting patients with advanced or terminal cancer. A 2016 survey [1] shows that the prevalence of moderate to severe pain in advanced, metastatic, or terminal cancer patients is $66.4 \%$. NHS England also estimates $5-15 \%$ of cancer patients [2] have inadequate pain control and to optimally manage many of these patients, interventions like nerve blocks, neurolysis and intrathecal morphine pumps are needed. In 2020, an article from the UK [2] showed that there was a large discrepancy between the need to perform a block and their implementation, with only 458 pain procedures performed for cancer patients in the financial year of 2018 - 2019. The distribution of these interventions is largely skewed, dependent on individual enthusiastic practitioners and their local set-ups. The European Pain Federation Task Force [3] provides

Manuscript submitted October 19, 2021, accepted November 12, 2021

Published online February 16, 2022

aPain Medicine, University Hospitals Plymouth NHS Trust, Plymouth, UK

${ }^{b}$ Chronic Pain Unit, Rowan House, Derriford Hospital, Plymouth, UK

${ }^{\mathrm{c} G P}$ Registrar, South West Deanery, Plymouth, UK

dPalliative Care, St Luke's Hospice Plymouth, Plymouth, UK

${ }^{e}$ Corresponding Author: Somnath Bagchi, Chronic Pain Unit, Rowan House,

Derriford Hospital, Plymouth, PL6 8BQ, UK. Email: s.bagchi@nhs.net

doi: https://doi.org/10.14740/jmc3800 a weak recommendation for neurolytic blocks but we argue that they can be useful in carefully selected patients [4-6]. Neurolysis involves the interruption of nociceptive transmission to the spinal cord from the peripheral tissues by destroying the nerve. The success or the range of clinical response of these blocks varies, and it may cause paresthesias and dysesthesias.

We work jointly with Palliative Care to identify patients struggling with pain control. Our standard approach to address severe opioid refractory pelvic pain in terminal patients is to perform intrathecal phenol neurolysis. We present a case here where this approach was not an option as the patient had spina bifida with a low lying conus ending at L5, cord tethering, and diastematomyelia.

\section{Case Report}

\section{Investigations}

The patient was a 45-year-old female patient born with spina bifida at T12-L1 and had undergone a thoracolumbar meningocele repair at 6 months of age. The operation was successful but left her with residual vertebral defect and soft tissue changes. The patient continued with her life and was fully functional with a full-time job, and had two cesarean sections under general anesthesia. The only chronic symptom was occasional pain in the lower back that radiated down the legs, left more than right, but she was not on any regular medication for this.

\section{Diagnosis}

The patient presented to clinicians with rectal bleeding in 2016 and was subsequently diagnosed as having an adenocarcinoma of her rectum involving the vaginal wall, and also a solitary lung metastasis. Treatment was initiated in the form of an anterior and posterior wall resection followed by pelvic radiation, microwave ablation of lung metastasis, and stereotactic radiation. Long courses of chemotherapy were also undertaken. Despite this, further imaging in early 2018 identified left-sided sacral spread with the invasion of the presacral soft tissue and sciatic nerve infiltration. The patient was beginning to suffer from pelvic pain and was subsequently commenced on a small 


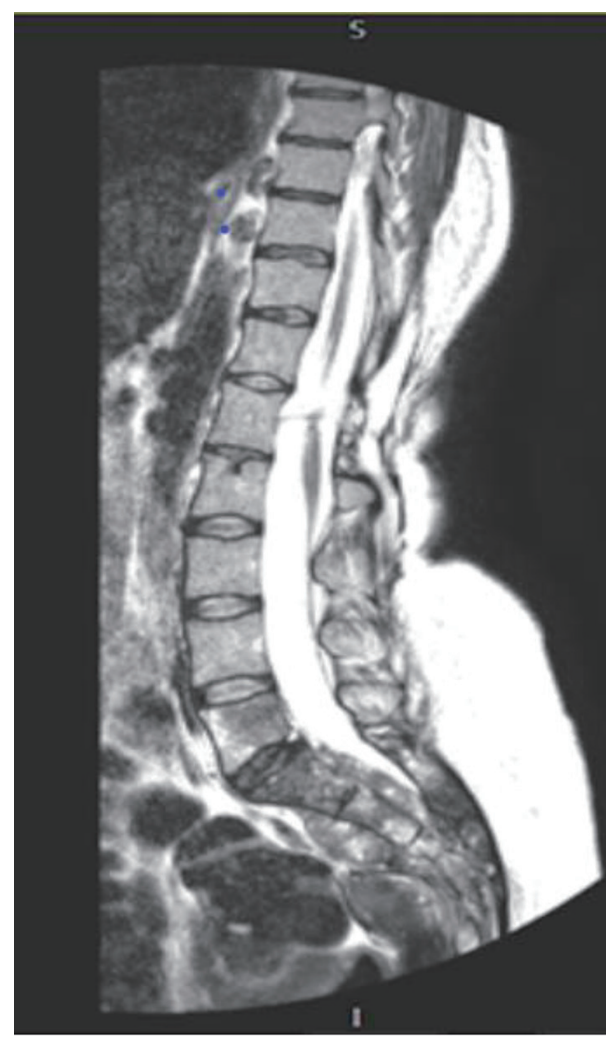

Figure 1. MRI of the lumber and sacral spine showing the metastatic deposit and the extent of the disease. MRI: magnetic resonance imaging.

dose of opioid (regular oxycodone modified release $10 \mathrm{mg}$ twice a day and oxycodone immediate release $5 \mathrm{mg}$ up to four times a day as necessary). Despite this, the patient was continuing to work with some limitations and seemed to be managing the pain with increases in her oral analgesia. Imaging in May 2019 identified the further progression of cancer and the patient decided against further chemotherapy offered by the oncologists. Emphasis was placed on symptomatic control. She developed increasing pain in the lower back radiating to the left leg, which was continuous burning and throbbing, resulting in further prescriptions of modified release oxycodone (oxycodone modified release $30 \mathrm{mg}$ twice daily in addition to pro re nata (prn) oxynorm liquid $10 \mathrm{mg}$ up to four times a day as prn), pregabalin (300 mg twice daily), celecoxib (200 mg twice daily), amitriptyline (50 $\mathrm{mg}$ once daily) and lidocaine (5\% lidocaine plaster, 1 plaster per day) plasters.

\section{Treatment}

In May 2020, the patient was admitted to the local hospice as an inpatient in an attempt to gain better control of her pain, which by now was severely impacting her quality of life. Most of the pain was in her pelvis and left leg, which was allodynic and hypersensitive. The pain was such that she was unable to get out of bed. During the 2 months admission at the hospice, several pharmacological cocktails were trialled including a

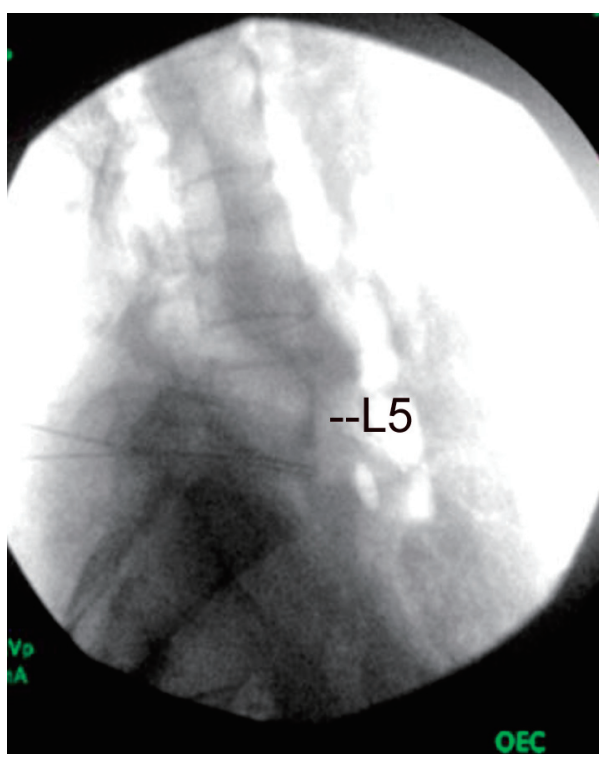

Figure 2. Lateral view of lumbar and sacral spine with needles in front of lower past of body of L5.

ketamine syringe driver, oxcarbazepine, lamotrigine, and clonazepam, without much success. Unfortunately, the patient's opiate dose needed to be reduced due to subsequent disorientation and opioid toxicity.

By the end of July 2020, the coronavirus disease 2019 (COVID-19)-related lockdown restrictions were slowly relaxed and at this point, the pain team was approached to consider an interventional option. After an Multidiciplinary Team Meeting discussion, a repeat magnetic resonance imaging (MRI) scan was done which showed intracerebral metastasis and further spread of the pelvic disease, with severe sacral spinal canal stenosis and involvement of perianal muscles on the left side (Fig. 1). With cerebral metastasis and deranged spinal anatomy, an intrathecal approach was ruled out and a superior hypogastric plexus (SHP) block was put forward as the optimum choice to address the pain [3, 5, 7]. The article by de Leno [8] demonstrated that such blocks provided satisfactory pain relief in $69 \%$ of patients with one of the outcome results being a $56 \%$ reduction in the mean opioid consumption. A diagnostic SHP block was therefore done using fluoroscopy via posterior approach with $5 \mathrm{~mL}$ of $0.25 \%$ chirocaine and 3.3 $\mathrm{mg}$ of dexamethasone. Following a successful local anesthetic block, which helped to reduce pain by $50 \%$ with the return of mild active movement of the left leg, a neurolytic SHP block was done 2 weeks later. Five milliliters of $6 \%$ aqueous phenol was used to do the neurolysis in the posterior classic approach (Figs. 2, 3).

The neurolytic intervention aimed to extend the period of pain relief demonstrated by the local anesthetic block, in an attempt to improve the patient's function in the hope that she could be discharged back to her residence. The patient had two children under 10 and was understandably desperate to return home so that she could spend her remaining time with her family. The procedure was performed under sedation in the prone position and under fluoroscopic control. The salient features 


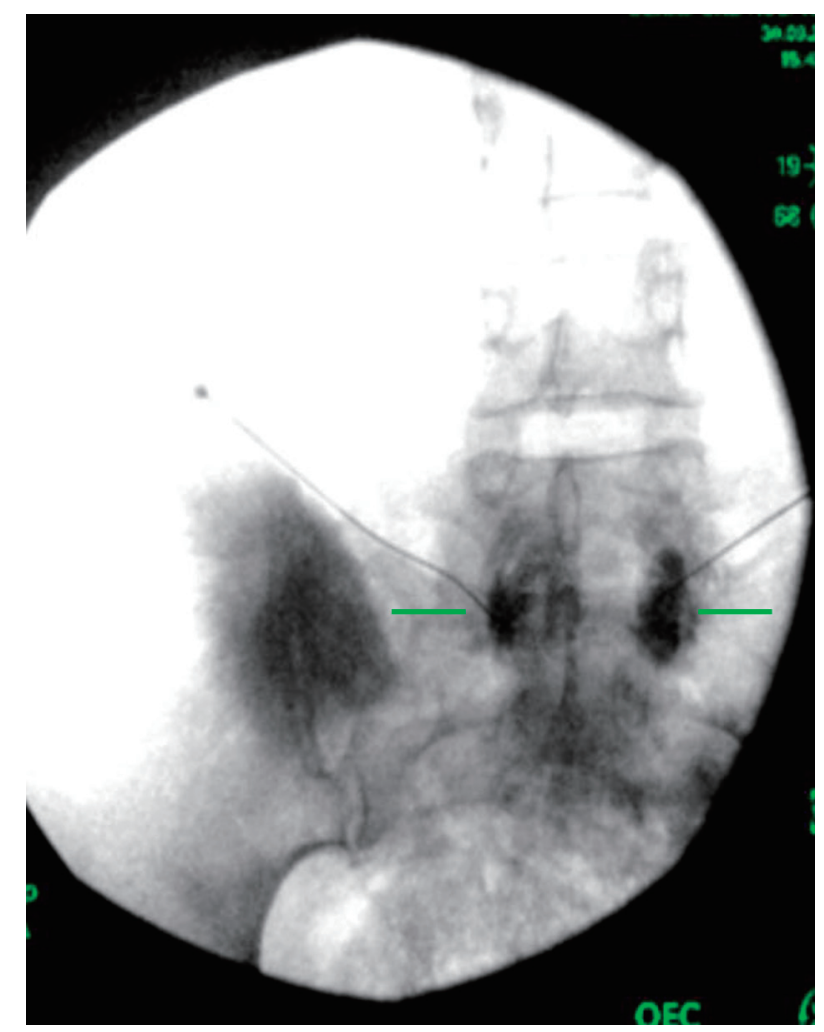

Figure 3. Fluoroscopic AP view with needle in position with contrast. Contrast (short lines) shows hypogastric plexus. AP: anterior-posterior.

are as follows: 1) The patient was provided with leaflets about neurolysis and explained the procedure to her and her partner, giving them adequate time to reflect upon it. 2) The common complications like hypotension, hypertension, puncture of the iliac vessel, infections, failure, and problems with positioning (prone) and sedation were explained. The rare complications were also mentioned like transient urinary incontinence, injury to the L5 nerve root, inadvertent puncture of pelvic viscera, and allergic reaction to drugs. 3) Any underlying psychological issues were addressed as these can have an impact on the perception of pain [9]. Co-existing anger, fear, depression and anxiety was addressed pre-procedure. The support workers and nurses at the hospice in our institution are very experienced in identifying and addressing these concerns and are actively involved in the holistic management of patient's wellbeing.

\section{Follow-up and outcomes}

Following the neurolysis, the patient reported a $70 \%$ reduction in her pain in the first 2 weeks, with a significant reduction in the sharp shooting pain in the legs, and complete resolution of the left leg hypersensitivity. This afforded a reduction in her amitriptyline which was reduced in stages from 50 to $10 \mathrm{mg}$. There was also a decrease in the frequency of prn opioid use and the background opioids decreased by $10 \%$, meaning that she had no further episodes of opioid toxicity. This reduction is very similar to various case reports. The series of 180 patients by Rocha et al [5] demonstrated that SHP neurolysis resulted in a sustained and significant visual analog scale (VAS) reduction by $49.55 \%$ with a reduction of opioid consumption of $12.55 \%$ at 3 months.

With a reduction in pain, the patient was noted to be much more positive and was able to work with her physiotherapists. Having been unable to get out of her bed before the intervention due to pain, she was able to progress to walking with a frame.

The progress made in optimizing her pain control and subsequent physical ability allowed for her to be discharged home safely. Despite the disease progression leading to a steep clinical deterioration once at home, the patient's pain remained well-controlled and resulted in a peaceful death at home, surrounded by her family after 10 weeks following the neurolysis.

\section{Discussion}

Cancer pain can be categorized into two main groups: tumormediated and treatment-related. They can also be further divided into nociceptive, neuropathic, or mixed, as well as being of either somatic or visceral origin. Nociceptive pain is caused by the stimulation of a normally functioning nervous system. Nociceptive somatic pain is often described as sharp and aching and is generally well localized. Nociceptive visceral pain, on the other hand, is typically caused by tumor growth, leading to stretching of visceral capsules, distension/obstruction of blood supply, or traction of the mesentery and is described as diffuse and poorly localized, cramping and aching pain, and often associated with autonomic dysfunction. Neuropathic pain is a result of damage or pathologic changes of the nervous system leading to abnormal neuronal activity. The pain is typically burning, tingling or electric shock-like. In the case presented above, the patient's pain was mixed, with both nociceptive and neuropathic components.

Neurolytic blocks are a relatively old technique and remain an important part of the armamentarium in the management of intractable cancer pain, especially from advanced cancer. There are case reports and series $[5,10,11]$ where multiple autonomic nerve plexuses are ablated to reduce pain, reduce opioid consumption and improve quality of life. As these are usually in form of case series at the best, the evidence rating of the neurolytic blocks is poor. But in carefully selected individuals, they can be beneficial when other modalities of pain relief are inadequate.

SHP neurolysis was first done by Plancarte et al [12] in 1990 and then repeated by de Leon Casasola et al with fluoroscopy in 1993 [8]. In our case, we used a similar classic posterior approach with fluoroscopy as described by de Leon et al. The main reason for adopting this approach was that it has a low incidence of complications [11] and the technique was familiar to our team. The posterior approach can, however, sometimes be difficult because of anatomic barriers such as the large transverse process of the L5 vertebra and the high arch of the iliac crest. In this case, we evaluated the computed tomography (CT) scan and MRI as part of the pre-procedure workup and did not feel that this problem would present an 
issue. A CT-guided approach can be useful in some cases with these difficulties [13]. Other approaches are described in the literature, such as a transvascular approach, transvaginal approach, transdiscal approach, an anterior approach [13]. The anterior approach to block the SHP carries a risk of infection as it goes through the bowel, and the transdiscal approach is associated with a potential risk of discitis, disk rupture or a disk herniation, so we avoided them. Cariat et al [14] did a comparative study of CT-guided anterior approach with the classic approach and reported that the CT-guided block had no serious side effects, despite potentially transversing the bowel, but had comparable pain relief, and we deduce that it affords no clinical benefit in patients without problematic anatomy as described above.

The narrow risk-benefit ratio associated with neurolysis techniques requires knowledge of anatomy to minimize undesirable effects [15]. The SHP is a retroperitoneal preaortic structure, localized at the level of the sacral promontory between the lower two-thirds of the L5 vertebral body and the upper third of the S1 vertebral body. It is just below the bifurcation of the aorta, lying close to the iliac vessels and the ureter in a fan-shaped pattern. The plexus is formed by two lateral roots and one medial root [16]. The lateral roots originate mainly from the lowest lumbar splanchnic nerves and contain a sympathetic component, while the medial root is a continuation of the abdominal aortic plexus from the inferior mesenteric plexus and has both sympathetic and parasympathetic components [17]. It is therefore a predominantly sympathetic plexus from the afferent tracts innervating the pelvic organs.

There is no clear consensus on patient selection, technique, or timing of these blocks. Patients with moderate to severe pain, not controlled with oral analgesics or having medication-related side effects, with a life expectancy of less than 6 months, are ideal candidates for the neurolysis [18]. The patient we present met these criteria, supporting neurolysis. The pain was severe, not managed by increasing analgesics, and the patient was thought to have a limited life expectancy. Unfortunately, these blocks should not be considered as measures to eliminate cancer pain because patients frequently experience co-existing somatic and neuropathic pain and as the disease progresses involve new structures and cause more pain. Therefore, oral pharmacologic therapy often needs to be continued, though the aim is to reduce the dose of medications and, in turn, reduce their side effects, allowing the patient to be more functional [19].

In our case, the chemical neurolytic agent used was $6 \%$ aqueous phenol. Compared to another commonly used neurolytic agent - absolute alcohol - the advantage of phenol is that it has local anesthetic properties, so is less painful on injection and has a lower risk of neuritis [20], although the intensity and duration of the block are marginally less than alcohol [19]. Phenol works by nonselective protein coagulation resulting in segmental demyelination and Wallerian degeneration at a concentration greater than $5 \%$. At a concentration of less than $5 \%$, phenol produces protein denaturation. There is a direct relationship between phenol concentration and the extent of nerve destruction. It acts as a local anesthetic agent at low concentration $(2 \%)$ and as a neurolytic agent at higher concentration
[21]. As in our case, a perineural injection produced a biphasic response with an initial local anesthetic effect (warm sensation) that leads to chronic denervation in the form of numbness due to the ongoing Wallerian degeneration which can continue for up to 14 days. The quality and extent of analgesia may fade slightly within the first $24 \mathrm{~h}$ and in this regard proper explanation to the patient is necessary.

We also recommend performing an initial diagnostic block to demonstrate the feasibility, efficacy and safety of the procedure. Many articles [22] support this practice of a diagnostic block with a local anesthetic before neurolysis to evaluate the impact of possible neurological deficit. In our case, the patient had a colostomy but had a functioning bladder, albeit requiring incontinence pads, but was extremely keen to avoid urinary catheterization. The diagnostic block demonstrated her ability to actively move her left leg and to tolerate bedding touching the leg, without significantly affecting her bladder function, and therefore gave her the confidence to go ahead with the neurolysis.

\section{Conclusion}

The success rate for SHP neurolysis for cancer pain varies between $60 \%$ and $70 \%$ in most case series and is very similar to our case with a reduction of pain of $60-65 \%$ at 2 months. The SHP neurolysis represents a reproducible and effective adjuvant in the management of pain in a carefully selected group of patients.

\section{Learning points}

Neurolytic blocks still remain an effective tool to manage intractable cancer pain but the patients should be carefully selected after informed consent and a careful assessment of risks and benefits.

\section{Acknowledgments}

None to declare.

\section{Financial Disclosure}

No grant or funding was available for the procedure or the preparation of the case report.

\section{Conflict of Interest}

None to declare.

\section{Informed Consent}

The patient had informed consent for the procedure. 


\section{Author Contributions}

Somnath Bagchi clinically reviewed the patient, consented her, did the procedure, did literature research and wrote the paper. Rosie Kemp collected the notes and followed the patient and helped in literature research. Christopher Green did the procedure jointly with Somnath Bagchi and provided expert knowledge to revise the writeup critically. Jeffrey Stephenson critically reviewed the paper and followed the patient up.

\section{Data Availability}

The authors declare that data supporting the findings of this study are available within the article.

\section{References}

1. van den Beuken-van Everdingen $\mathrm{MH}$, Hochstenbach LM, Joosten EA, Tjan-Heijnen VC, Janssen DJ. Update on prevalence of pain in patients with cancer: systematic review and meta-analysis. J Pain Symptom Manage. 2016;51(6):1070-1090.e9.

2. Duarte RV, Sale A, Desai P, Marshall T, Eldabe S. The unmet need for intrathecal drug delivery pumps for the treatment of cancer pain in England: an assessment of the hospital episode statistics database. Neuromodulation. 2020;23(7):1029-1033.

3. Bennett MI, Eisenberg E, Ahmedzai SH, Bhaskar A, O'Brien T, Mercadante S, Krcevski Skvarc N, et al. Standards for the management of cancer-related pain across Europe - A position paper from the EFIC task force on cancer pain. Eur J Pain. 2019;23(4):660-668.

4. Candido KD, Kusper TM, Knezevic NN. New cancer pain treatment options. Curr Pain Headache Rep. 2017;21(2):12.

5. Rocha A, Plancarte R, Nataren RGR, Carrera IHS, Pacheco V, Hernandez-Porras BC. Effectiveness of superior hypogastric plexus neurolysis for pelvic cancer pain. Pain Physician. 2020;23(2):203-208.

6. Plancarte-Sanchez R, Guajardo-Rosas J, Guillen-Nunez R. Superior hypogastric plexus block and ganglion impar. Tech Reg Anesth Pain Manag. 2005;9:86-90.

7. Van Zundert J, Hartrick C, Patijn J, Huygen F, Mekhail $\mathrm{N}$, van Kleef $\mathrm{M}$. Evidence-based interventional pain medicine according to clinical diagnoses. Pain Pract. 2011;11(5):423-429.

8. de Leon-Casasola OA, Kent E, Lema MJ. Neurolytic superior hypogastric plexus block for chronic pelvic pain associated with cancer. Pain. 1993;54(2):145-151.
9. Cleeland CS. The impact of pain on the patient with cancer. Cancer. 1984;54(11 Suppl):2635-2641.

10. Mercadante S. The combination of superior hypogastric plexus block and the block of the ganglium impair in a patient with abdominal and perineal pain poorly responsive to opioids. J Pain Symptom Manage. 2019;58(1):e5-e8.

11. Plancarte R, de Leon-Casasola OA, El-Helaly M, Allende S, Lema MJ. Neurolytic superior hypogastric plexus block for chronic pelvic pain associated with cancer. Reg Anesth. 1997;22(6):562-568.

12. Plancarte R, Amescua C, Patt RB, Aldrete JA. Superior hypogastric plexus block for pelvic cancer pain. Anesthesiology. 1990;73(2):236-239.

13. Ghoneim AA, Mansour SM. Comparative study between computed tomography guided superior hypogastric plexus block and the classic posterior approach: A prospective randomized study. Saudi J Anaesth. 2014;8(3):378383.

14. Cariati M, De Martini G, Pretolesi F, Roy MT. CT-guided superior hypogastric plexus block. J Comput Assist Tomogr. 2002;26(3):428-431.

15. de Leon-Casasola OA. Critical evaluation of chemical neurolysis of the sympathetic axis for cancer pain. Cancer Control. 2000;7(2):142-148.

16. Paraskevas G, Tsitsopoulos P, Papaziogas B, Natsis K, Martoglou S, Stoltidou A, Kitsoulis P. Variability in superior hypogastric plexus morphology and its clinical applications: a cadaveric study. Surg Radiol Anat. 2008;30(6):481-488.

17. Kim H, Nam YS, Lee UY, Kim IB, Kim YS. Anatomy of the superior hypogastric plexus and its application in nerve-sparing paraaortic lymphadenectomy. Folia Morphol (Warsz). 2021;80(1):70-75.

18. Koyyalagunta D, Burton AW. The role of chemical neurolysis in cancer pain. Curr Pain Headache Rep. 2010;14(4):261-267.

19. Burton AW, Phan PC, Cousins MJ. Treatment of cancer pain: role of neural blockade and neuromodulation. In: Cousins MJ, Carr DB, Horlocker TT, Bridenbaugh PO. NeuraBlockade in clinical anesthesia and pain medicine, 4th edn. Philadelphia: Lippincott Williams and Wilkins; 2009:1111-1153.

20. Part RB, Cousins MJ. Techniques for neurolytic neural blockade. In: Cousins MJ. Bridenbaugh PO (Eds): Neural blockade in clinical anesthesia and management of pain. 2nd ed, Philadelphia. Lippincon-Raven. 1998. p. 10071061.

21. Wood KM. The use of phenol as a neurolytic agent: a review. Pain. 1978;5(3):205-229.

22. Teng J. Cancer pain and neurolysis: seminars in anesthesia. Perioperative Medicine and Pain. 2003;22(3):175185. 